\title{
Local Fractional Laplace Variational Iteration Method for Solving Linear Partial Differential Equations with Local Fractional Derivative
}

\author{
Ai-Min Yang, ${ }^{1,2}$ Jie Li, ${ }^{1,3}$ H. M. Srivastava, ${ }^{4}$ Gong-Nan Xie, ${ }^{5}$ and Xiao-Jun Yang ${ }^{6}$ \\ ${ }^{1}$ College of Science, Hebei United University, Tangshan 063009, China \\ ${ }^{2}$ College of Mechanical Engineering, Yanshan University, Qinhuangdao 066004, China \\ ${ }^{3}$ School of Materials and Metallurgy, Northeast University, Shenyang 110819, China \\ ${ }^{4}$ Department of Mathematics and Statistics, University of Victoria, Victoria, BC, Canada V8W 3R4 \\ ${ }^{5}$ School of Mechanical Engineering, Northwestern Polytechnical University, Xian, Shaanxi 710048, China \\ ${ }^{6}$ Department of Mathematics and Mechanics, China University of Mining and Technology, Xuzhou, Jiangsu 221008, China
}

Correspondence should be addressed to Xiao-Jun Yang; dyangxiaojun@163.com

Received 4 April 2014; Accepted 28 April 2014; Published 17 July 2014

Academic Editor: Bing Xu

Copyright (C) 2014 Ai-Min Yang et al. This is an open access article distributed under the Creative Commons Attribution License, which permits unrestricted use, distribution, and reproduction in any medium, provided the original work is properly cited.

\begin{abstract}
The local fractional Laplace variational iteration method was applied to solve the linear local fractional partial differential equations. The local fractional Laplace variational iteration method is coupled by the local fractional variational iteration method and Laplace transform. The nondifferentiable approximate solutions are obtained and their graphs are also shown.
\end{abstract}

\section{Introduction}

Fractional calculus [1] has successfully been used to study the mathematical and physical problems arising in science and engineering. Fractional differential equations are applied to describe the dynamical systems in physics and engineering (see $[2,3])$. It is one of the hot topics for finding the solutions for the fractional differential equations for scientists and engineers. There are many analytical and numerical methods for solving them, such as the spectral LegendreGauss-Lobatto collocation method [4], the shifted JacobiGauss-Lobatto collocation method [5], the variation iteration method [6], the heat-balance integral method [7], the Adomian decomposition method [8], the finite element method [9], and the finite difference method [10].

The above methods did not deal with some nondifferentiable problems arising in mathematics and physics (see [11-13]). Local fractional calculus (see [12-14] and the cited references) is the best choice to deal with them. Some methods for solving the local fractional differential equations were suggested, such as the Cantor-type cylindricalcoordinate method [15], the local fractional variational iteration method [16, 17], the local fractional decomposition method [18], the local fractional series expansion method [19], the local fractional Laplace transform method [20], and local fractional function decomposition method [21, 22]. More recently, the coupling schemes for local fractional variational iteration method and Laplace transform were suggested in [23]. However, the results are very little. In this paper, our aim is to use the local fractional Laplace variational iteration method to solve the linear local fractional partial differential equations. The structure of the paper is suggested as follows. In Section 2 the basic theory of local fractional calculus and local fractional Laplace transform are introduced. Section 3 is devoted to the local fractional Laplace variational iteration method. In Section 4, the four examples for the local fractional partial differential equations are given. Finally, the conclusions are considered in Section 5. 


\section{Local Fractional Calculus and Local Fractional Laplace Transform}

In this section, we present the basic theory of local fractional calculus and concepts of local fractional Laplace transform.

The local fractional derivative of $f(x)$ of order $\alpha$ is defined as $[12-15]$

$$
\frac{d^{\alpha} f\left(x_{0}\right)}{d x^{\alpha}}=\frac{\Delta^{\alpha}\left(f(x)-f\left(x_{0}\right)\right)}{\left(x-x_{0}\right)^{\alpha}},
$$

where

$$
\Delta^{\alpha}\left(f(x)-f\left(x_{0}\right)\right) \cong \Gamma(1+\alpha)\left[f(x)-f\left(x_{0}\right)\right] .
$$

The local fractional integral of $f(x)$ of order $\alpha$ in the interval $[a, b]$ is defined as $[12-14,16-23]$

$$
\begin{aligned}
a_{b}^{(\alpha)} f(x) & =\frac{1}{\Gamma(1+\alpha)} \int_{a}^{b} f(t)(d t)^{\alpha} \\
& =\frac{1}{\Gamma(1+\alpha)} \lim _{\Delta t \rightarrow 0} \sum_{j=0}^{j=N-1} f\left(t_{j}\right)\left(\Delta t_{j}\right)^{\alpha},
\end{aligned}
$$

where the partitions of the interval $[a, b]$ are $\left(t_{j}, t_{j+1}\right)$, with $\Delta t_{j}=t_{j+1}-t_{j}, t_{0}=a, t_{N}=b$, and $\Delta t=\max \left\{\Delta t_{0}, \Delta t_{1}, \Delta t_{j}, \ldots\right\}$, $j=0, \ldots, N-1$.

The local fractional series of nondifferentiable function used in this paper are presented as follows [12-14]:

$$
\begin{gathered}
E_{\alpha}\left(x^{\alpha}\right)=\sum_{k=0}^{\infty} \frac{x^{\alpha k}}{\Gamma(1+k \alpha)}, \\
\sin _{\alpha}\left(x^{\alpha}\right)=\sum_{k=0}^{\infty}(-1)^{k} \frac{x^{(2 k+1) \alpha}}{\Gamma[1+(2 k+1) \alpha]} \\
\cos _{\alpha}\left(x^{\alpha}\right)=\sum_{k=0}^{\infty}(-1)^{k} \frac{x^{2 \alpha k}}{\Gamma(1+2 \alpha k)} \\
\sinh _{\alpha}\left(x^{\alpha}\right)=\sum_{k=0}^{\infty} \frac{x^{(2 k+1) \alpha}}{\Gamma[1+(2 k+1) \alpha]} \\
\cosh _{\alpha}\left(x^{\alpha}\right)=\sum_{k=0}^{\infty} \frac{x^{2 \alpha k}}{\Gamma(1+2 \alpha k)} .
\end{gathered}
$$

The properties of local fractional derivatives and integral of nondifferentiable functions are given by $[12,13]$

$$
\begin{aligned}
\frac{d^{\alpha}}{d x^{\alpha}} \frac{x^{n \alpha}}{\Gamma(1+n \alpha)}= & \frac{x^{(n-1) \alpha}}{\Gamma(1+(n-1) \alpha)}, \\
\frac{d^{\alpha}}{d x^{\alpha}} E_{\alpha}\left(x^{\alpha}\right) & =E_{\alpha}\left(x^{\alpha}\right), \\
\frac{d^{\alpha}}{d x^{\alpha}} \sin _{\alpha}\left(x^{\alpha}\right) & =\cos _{\alpha}\left(x^{\alpha}\right), \\
\frac{d^{\alpha}}{d x^{\alpha}} \cos _{\alpha}\left(x^{\alpha}\right) & =-\sin _{\alpha}\left(x^{\alpha}\right),
\end{aligned}
$$

$$
\begin{gathered}
\frac{d^{\alpha}}{d x^{\alpha}} \sinh _{\alpha}\left(x^{\alpha}\right)=\cosh _{\alpha}\left(x^{\alpha}\right), \\
\frac{d^{\alpha}}{d x^{\alpha}} \cosh _{\alpha}\left(x^{\alpha}\right)=-\sinh _{\alpha}\left(x^{\alpha}\right), \\
{ }_{0} I_{x}^{(\alpha)} \frac{x^{n \alpha}}{\Gamma(1+n \alpha)}=\frac{x^{(n+1) \alpha}}{\Gamma(1+(n+1) \alpha)} .
\end{gathered}
$$

The local fractional Laplace transform is defined as [12, 2022]

$$
\begin{aligned}
\widetilde{L}_{\alpha}\{f(x)\}= & f_{s}^{\widetilde{L}, \alpha}(s) \\
= & \frac{1}{\Gamma(1+\alpha)} \\
& \times \int_{0}^{\infty} E_{\alpha}\left(-s^{\alpha} x^{\alpha}\right) f(x)(d x)^{\alpha}, \quad 0<\alpha \leq 1,
\end{aligned}
$$

where $f(x)$ is local fractional continuous and $s^{\alpha}=\beta^{\alpha}+i^{\alpha} \infty^{\alpha}$.

The inverse formula of local fractional Laplace transform is defined as [12, 20-22]

$$
\begin{aligned}
f(x) & =\widetilde{L}_{\alpha}^{-1}\left\{f_{s}^{L, \alpha}(s)\right\} \\
& =\frac{1}{(2 \pi)^{\alpha}} \int_{\beta-i \infty}^{\beta+i \infty} E_{\alpha}\left(s^{\alpha} x^{\alpha}\right) f_{s}^{\widetilde{L}, \alpha}(s)(d s)^{\alpha},
\end{aligned}
$$

where $s^{\alpha}=\beta^{\alpha}+i^{\alpha} \infty^{\alpha}$ and $\operatorname{Re}\left(s^{\alpha}\right)=\beta^{\alpha}$.

The local fractional convolution of two functions is defined as $[12,20-22]$

$$
f_{1}(x) * f_{2}(x)=\frac{1}{\Gamma(1+\alpha)} \int_{-\infty}^{\infty} f_{1}(t) f_{2}(x-t)(d t)^{\alpha} .
$$

The properties for local fractional Laplace transform used in the paper are given as [12]

$$
\begin{gathered}
\widetilde{L}_{\alpha}\{a f(x)+b g(x)\}=a \widetilde{L}_{\alpha}\{f(x)\}+b \widetilde{L}_{\alpha}\{g(x)\}, \\
\widetilde{L}_{\alpha}\left\{f^{(n \alpha)}(x)\right\}=s^{n \alpha} \widetilde{L}_{\alpha}\{f(x)\} \\
-\sum_{k=1}^{n} s^{(k-1) \alpha} f^{(n-k) \alpha}(0), \\
F_{\alpha}\left\{f_{1}(x) * f_{2}(x)\right\}=f_{\omega, 1}^{F, \alpha}(\omega) f_{\omega, 2}^{F, \alpha}(\omega), \\
\widetilde{L}_{\alpha}\left\{\sin _{\alpha}\left(c^{\alpha} x^{\alpha}\right)\right\}=\frac{c^{\alpha}}{s^{2 \alpha}+c^{2 \alpha}}, \\
\tilde{L}_{\alpha}\left\{\cos _{\alpha}\left(c^{\alpha} x^{\alpha}\right)\right\}=\frac{s^{\alpha}}{s^{2 \alpha}+c^{2 \alpha}}, \\
\widetilde{L}_{\alpha}\left\{x^{k \alpha}\right\}=\frac{\Gamma(1+k \alpha)}{s^{(k+1) \alpha}} .
\end{gathered}
$$

\section{Analysis of the Method}

In this section, we introduce the idea of local fractional variational iteration method $[16,17]$, which is coupled by 
the local fractional variational iteration method and Laplace transform.

Let us consider the following nonlinear operator with local fractional derivative:

$$
L_{\alpha} u-N_{\alpha} u=0,
$$

where the linear local fractional differential operator denotes $L_{\alpha}=\left(d^{k \alpha} / d s^{k \alpha}\right)$ and $u(x)$ is a source term of the nondifferential function.

Following the local fractional Laplace variational iteration method [23], we have the local fractional functional formula as follows:

$$
u_{n+1}(x)=u_{n}(x)+{ }_{0} I_{x}^{(\alpha)}\left\{\frac{\lambda(t)^{\alpha}}{\Gamma(1+\alpha)}\left[L_{\alpha} u_{n}(t)-N_{\alpha} u_{n}\right]\right\},
$$

which leads to

$$
u_{n+1}(x)=u_{n}(x)+{ }_{0} I_{x}^{(\alpha)}\left\{\frac{\lambda(x-t)^{\alpha}}{\Gamma(1+\alpha)}\left[L_{\alpha} u_{n}(t)-N_{\alpha} u_{n}\right]\right\} \text {. }
$$

Using the local fractional Laplace transform, from (12), we get $\widetilde{L}_{\alpha}\left\{u_{n+1}(x)\right\}=\widetilde{L}_{\alpha}\left\{u_{n}(x)\right\}$

$$
+\tilde{L}_{\alpha}\left\{\frac{\lambda(x)^{\alpha}}{\Gamma(1+\alpha)}\right\} \widetilde{L}_{\alpha}\left\{L_{\alpha} u_{n}(x)-N_{\alpha} u_{n}(x)\right\} .
$$

Taking the local fractional variation [21], we obtain

$$
\begin{aligned}
\delta^{\alpha}\left\{\widetilde{L}_{\alpha}\left\{u_{n+1}(x)\right\}\right\} \\
=\delta^{\alpha}\left\{\widetilde{L}_{\alpha}\left\{u_{n}(x)\right\}\right\} \\
\quad+\delta^{\alpha}\left\{\widetilde{L}_{\alpha}\left\{\frac{\lambda(x)^{\alpha}}{\Gamma(1+\alpha)}\right\} \widetilde{L}_{\alpha}\left\{L_{\alpha} u_{n}(x)-N_{\alpha} u_{n}(x)\right\}\right\}
\end{aligned}
$$

which leads to

$$
\begin{aligned}
\delta^{\alpha}\left\{\widetilde{L}_{\alpha}\left\{u_{n+1}(x)\right\}\right\}= & \delta^{\alpha}\left\{\widetilde{L}_{\alpha}\left\{u_{n}(x)\right\}\right\} \\
& +\widetilde{L}_{\alpha}\left\{\frac{\lambda(x)^{\alpha}}{\Gamma(1+\alpha)}\right\}\left\{\delta^{\alpha}\left\{\widetilde{L}_{\alpha}\left\{L_{\alpha} u_{n}(x)\right\}\right\}\right\} \\
= & 0,
\end{aligned}
$$

where

$$
\begin{aligned}
\widetilde{L}_{\alpha}\left\{L_{\alpha} u_{n}(x)\right\}= & s^{k \alpha} \widetilde{L}_{\alpha}\left\{u_{n}(x)\right\}-s^{(k-1) \alpha} u_{n}(0) \\
& -s^{(k-2) \alpha} u_{n}^{(\alpha)}(0)-\cdots-u_{n}^{((k-1) \alpha)}(0) \\
= & s^{k \alpha} \widetilde{L}_{\alpha}\left\{u_{n}(x)\right\} .
\end{aligned}
$$

Hence, from (15) and (16) we get

$$
1+\widetilde{L}_{\alpha}\left\{\frac{\lambda(x)^{\alpha}}{\Gamma(1+\alpha)}\right\} s^{k \alpha}=0,
$$

which yields

$$
\widetilde{L}_{\alpha}\left\{\frac{\lambda(x)^{\alpha}}{\Gamma(1+\alpha)}\right\}=-\frac{1}{s^{k \alpha}} .
$$

Hence, we get the new iteration algorithm as follows:

$$
\begin{aligned}
\widetilde{L}_{\alpha}\left\{u_{n+1}(x)\right\}= & \widetilde{L}_{\alpha}\left\{u_{n}(x)\right\} \\
& -\frac{1}{s^{k \alpha}} \widetilde{L}_{\alpha}\left\{\left(L_{\alpha} u_{n}(x)-N_{\alpha} u_{n}(x)\right)\right\} \\
= & \widetilde{L}_{\alpha}\left\{u_{n}(x)\right\}-\frac{1}{s^{k \alpha}} \widetilde{L}_{\alpha}\left\{L_{\alpha} u_{n}(x)\right\} \\
& +\frac{1}{s^{k \alpha}} \widetilde{L}_{\alpha}\left\{N_{\alpha} u_{n}(x)\right\},
\end{aligned}
$$

where the initial value is suggested as

$$
\frac{s^{(k-1) \alpha} u(0)+s^{(k-2) \alpha} u^{(\alpha)}(0)+\cdots+u^{((k-1) \alpha)}(0)}{s^{k \alpha}}=0 .
$$

Therefore, we have the local fractional series solution of (10)

$$
\widetilde{L}_{\alpha}\{u\}=\lim _{n \rightarrow \infty} \widetilde{L}_{\alpha}\left\{u_{n}\right\}
$$

so that

$$
u=\lim _{n \rightarrow \infty} \widetilde{L}_{\alpha}^{-1}\left\{\widetilde{L}_{\alpha} u_{n}\right\}
$$

The above process is called the local fractional variational iteration method.

\section{The Nondifferentiable Solutions for Linear Local Fractional Differential Equations}

In this section, we present the examples for linear local fractional differential equations of high order.

Example 1. The local fractional differential equation is presented as

$$
\frac{\partial^{3 \alpha} u(x, t)}{\partial t^{3 \alpha}}=\frac{\partial^{2 \alpha} u(x, t)}{\partial x^{2 \alpha}}
$$

subject to the initial value

$$
\frac{\partial^{\alpha} u(0, t)}{\partial x^{\alpha}}=0, \quad u(0, t)=E_{\alpha}\left(-t^{\alpha}\right) .
$$


From (19) and (23) we obtain

$$
\begin{aligned}
\widetilde{L}_{\alpha}\left\{u_{n+1}(x, t)\right\} \\
=\widetilde{L}_{\alpha}\left\{u_{n}(x, t)\right\} \\
\quad-\frac{1}{s^{2 \alpha}} \widetilde{L}_{\alpha}\left\{\frac{\partial^{2 \alpha} u_{n}(x, t)}{\partial x^{2 \alpha}}-\frac{\partial^{3 \alpha} u_{n}(x, t)}{\partial t^{3 \alpha}}\right\} \\
=\widetilde{L}_{\alpha}\left\{u_{n}(x, t)\right\} \\
\quad-\frac{1}{s^{2 \alpha}}\left\{s^{2 \alpha} u_{n}(s, t)-s^{\alpha} u_{n}(0, t)-u_{n}^{(\alpha)}(0, t)\right. \\
=\widetilde{L}_{\alpha}\left\{u_{n}(x, t)\right\} \\
\quad-\frac{1}{s^{2 \alpha}}\left\{s^{2 \alpha} u_{n}(s, t)-s^{\alpha} u_{n}(0, t)-\frac{\partial^{3 \alpha} u_{n}(s, t)}{\partial t^{3 \alpha}}\right\}
\end{aligned}
$$

where the initial value is given by

$$
\widetilde{L}_{\alpha}\left\{u_{0}(x, t)\right\}=u_{0}(s, t)=\widetilde{L}_{\alpha}\left\{E_{\alpha}\left(-t^{\alpha}\right)\right\}=\frac{E_{\alpha}\left(-t^{\alpha}\right)}{s^{\alpha}}
$$

Therefore, the successive approximations are

$$
\begin{aligned}
& \widetilde{L}_{\alpha}\left\{u_{1}(x, t)\right\} \\
&=\tilde{L}_{\alpha}\left\{u_{0}(x, t)\right\} \\
&-\frac{1}{s^{2 \alpha}}\left\{s^{2 \alpha} u_{0}(s, t)-s^{\alpha} u_{0}(0, t)-\frac{\partial^{3 \alpha} u_{0}(s, t)}{\partial t^{3 \alpha}}\right\} \\
&= \frac{E_{\alpha}\left(-t^{\alpha}\right)}{s^{\alpha}}-\frac{E_{\alpha}\left(-t^{\alpha}\right)}{s^{3 \alpha}} \\
&= E_{\alpha}\left(-t^{\alpha}\right) \sum_{k=0}^{1}(-1)^{k} \frac{1}{s^{(2 k+1) \alpha}},
\end{aligned}
$$$$
\tilde{L}_{\alpha}\left\{u_{2}(x, t)\right\}
$$$$
=\widetilde{L}_{\alpha}\left\{u_{1}(x, t)\right\}
$$$$
-\frac{1}{s^{2 \alpha}}\left\{s^{2 \alpha} u_{1}(s, t)-s^{\alpha} u_{1}(0, t)-\frac{\partial^{3 \alpha} u_{1}(s, t)}{\partial t^{3 \alpha}}\right\}
$$$$
=\frac{E_{\alpha}\left(-t^{\alpha}\right)}{s^{\alpha}}-\frac{E_{\alpha}\left(-t^{\alpha}\right)}{s^{3 \alpha}}+\frac{E_{\alpha}\left(-t^{\alpha}\right)}{s^{5 \alpha}}
$$$$
=E_{\alpha}\left(-t^{\alpha}\right) \sum_{k=0}^{2}(-1)^{k} \frac{1}{s^{(2 k+1) \alpha}} \text {, }
$$

$$
\begin{aligned}
& \widetilde{L}_{\alpha}\{\left.u_{3}(x, t)\right\} \\
&= \widetilde{L}_{\alpha}\left\{u_{2}(x, t)\right\} \\
&-\frac{1}{s^{2 \alpha}}\left\{s^{2 \alpha} u_{2}(s, t)-s^{\alpha} u_{2}(0, t)-\frac{\partial^{3 \alpha} u_{2}(s, t)}{\partial t^{3 \alpha}}\right\} \\
&= \frac{E_{\alpha}\left(-t^{\alpha}\right)}{s^{\alpha}}-\frac{E_{\alpha}\left(-t^{\alpha}\right)}{s^{3 \alpha}}+\frac{E_{\alpha}\left(-t^{\alpha}\right)}{s^{5 \alpha}}-\frac{E_{\alpha}\left(-t^{\alpha}\right)}{s^{7 \alpha}} \\
&= E_{\alpha}\left(-t^{\alpha}\right) \sum_{k=0}^{3}(-1)^{k} \frac{1}{s^{(2 k+1) \alpha}}, \\
& \widetilde{L}_{\alpha}\left\{u_{4}(x, t)\right\} \\
&=\widetilde{L}_{\alpha}\left\{u_{3}(x, t)\right\} \\
&-\frac{1}{s^{2 \alpha}}\left\{s^{2 \alpha} u_{3}(s, t)-s^{\alpha} u_{3}(0, t)-\frac{\partial^{3 \alpha} u_{3}(s, t)}{\partial t^{3 \alpha}}\right\} \\
&= \frac{E_{\alpha}\left(-t^{\alpha}\right)}{s^{\alpha}}-\frac{E_{\alpha}\left(-t^{\alpha}\right)}{s^{3 \alpha}}+\frac{E_{\alpha}\left(-t^{\alpha}\right)}{s^{5 \alpha}} \\
&-\frac{E_{\alpha}\left(-t^{\alpha}\right)}{s^{7 \alpha}}+\frac{E_{\alpha}\left(-t^{\alpha}\right)}{s^{9 \alpha}} \\
& E_{\alpha}\left(-t^{\alpha}\right) \sum_{k=0}^{4}(-1)^{k} \frac{1}{s^{(2 k+1) \alpha}},
\end{aligned}
$$

$\vdots$

$$
\begin{aligned}
& \tilde{L}_{\alpha}\left\{u_{n}(x, t)\right\} \\
& =\tilde{L}_{\alpha}\left\{u_{n-1}(x, t)\right\} \\
& \quad-\frac{1}{s^{2 \alpha}}\left\{s^{2 \alpha} u_{n-1}(s, t)-s^{\alpha} u_{n-1}(0, t)\right. \\
& \left.\quad-\frac{\partial^{3 \alpha} u_{n-1}(s, t)}{\partial t^{3 \alpha}}\right\} \\
& =E_{\alpha}\left(-t^{\alpha}\right) \sum_{k=0}^{n}(-1)^{k} \frac{1}{s^{(2 k+1) \alpha}} .
\end{aligned}
$$

Hence, we get

$$
\begin{aligned}
u(x, t) & =\lim _{n \rightarrow \infty} \widetilde{L}_{\alpha}^{-1}\left\{\widetilde{L}_{\alpha} u_{n}\right\} \\
& =\lim _{n \rightarrow \infty} \widetilde{L}_{\alpha}^{-1}\left\{E_{\alpha}\left(-t^{\alpha}\right) \sum_{k=0}^{n}(-1)^{i} \frac{1}{s^{(2 k+1) \alpha}}\right\} \\
& =E_{\alpha}\left(-t^{\alpha}\right) \sum_{k=0}^{n} \frac{(-1)^{k} x^{2 k \alpha}}{\Gamma(1+2 k \alpha)} \\
& =E_{\alpha}\left(-t^{\alpha}\right) \cos _{\alpha}\left(x^{\alpha}\right)
\end{aligned}
$$

and its graph is shown in Figure 1. 


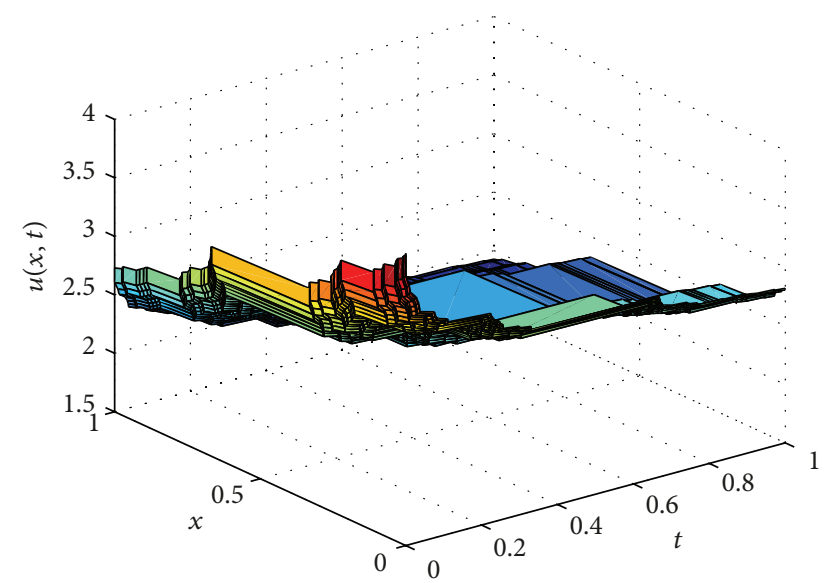

Figure 1: Plot of $u(x, t)$ with the fractal dimension $\alpha=\ln 2 / \ln 3$.

Example 2. We report the following local fractional partial differential equation:

$$
\frac{\partial^{2 \alpha} u(x, t)}{\partial t^{2 \alpha}}=\frac{\partial^{3 \alpha} u(x, t)}{\partial x^{3 \alpha}}
$$

The initial value is given by

$$
\frac{\partial^{2 \alpha} u(0, t)}{\partial x^{2 \alpha}}=0, \quad \frac{\partial^{\alpha} u(0, t)}{\partial x^{\alpha}}=E_{\alpha}\left(t^{\alpha}\right), \quad u(0, t)=0 .
$$

In view of (19) and (29) the local fractional iteration algorithm can be written as follows:

$$
\begin{aligned}
\widetilde{L}_{\alpha}\left\{u_{n+1}(x, t)\right\} \\
=\widetilde{L}_{\alpha}\left\{u_{n}(x, t)\right\} \\
\quad-\frac{1}{s^{3 \alpha}} \widetilde{L}_{\alpha}\left\{\frac{\partial^{3 \alpha} u_{n}(x, t)}{\partial x^{3 \alpha}}-\frac{\partial^{2 \alpha} u_{n}(x, t)}{\partial t^{2 \alpha}}\right\} \\
=\widetilde{L}_{\alpha}\left\{u_{n}(x, t)\right\} \\
-\frac{1}{s^{3 \alpha}}\left\{s^{3 \alpha} u_{n}(s, t)-s^{2 \alpha} u_{n}(0, t)-s^{\alpha} u_{n}^{(\alpha)}(0, t)\right. \\
\left.\quad-u_{n}^{(2 \alpha)}(0, t)-\frac{\partial^{3 \alpha} u_{n}(s, t)}{\partial t^{3 \alpha}}\right\} \\
=\widetilde{L}_{\alpha}\left\{u_{n}(x, t)\right\} \\
\quad-\frac{1}{s^{3 \alpha}}\left\{s^{3 \alpha} u_{n}(s, t)-s^{\alpha} u_{n}^{(\alpha)}(0, t)-\frac{\partial^{3 \alpha} u_{n}(s, t)}{\partial t^{3 \alpha}}\right\},
\end{aligned}
$$

where the initial value is

$$
\begin{aligned}
\tilde{L}_{\alpha}\left\{u_{0}(x, t)\right\} & =u_{0}(s, t) \\
& =\tilde{L}_{\alpha}\left\{\frac{x^{\alpha}}{\Gamma(1+\alpha)} E_{\alpha}\left(t^{\alpha}\right)\right\}=\frac{E_{\alpha}\left(t^{\alpha}\right)}{s^{2 \alpha}} .
\end{aligned}
$$

Making use of (31) and (32), the successive approximate solutions are shown as follows:

$$
\begin{aligned}
& \widetilde{L}_{\alpha}\left\{u_{1}(x, t)\right\} \\
& =\widetilde{L}_{\alpha}\left\{u_{0}(x, t)\right\} \\
& -\frac{1}{s^{3 \alpha}}\left\{s^{3 \alpha} u_{0}(s, t)-s^{\alpha} u_{0}^{(\alpha)}(0, t)-\frac{\partial^{3 \alpha} u_{0}(s, t)}{\partial t^{3 \alpha}}\right\} \\
& =\widetilde{L}_{\alpha}\left\{u_{0}(x, t)\right\} \\
& -\frac{1}{s^{3 \alpha}}\left\{s^{3 \alpha} u_{0}(s, t)-s^{\alpha} E_{\alpha}\left(t^{\alpha}\right)-\frac{\partial^{3 \alpha} u_{0}(s, t)}{\partial t^{3 \alpha}}\right\} \\
& =\frac{E_{\alpha}\left(t^{\alpha}\right)}{s^{2 \alpha}}+\frac{E_{\alpha}\left(t^{\alpha}\right)}{s^{5 \alpha}} \\
& =E_{\alpha}\left(t^{\alpha}\right) \sum_{k=0}^{1} \frac{1}{s^{(3 k+2) \alpha}}, \\
& \widetilde{L}_{\alpha}\left\{u_{2}(x, t)\right\} \\
& =\widetilde{L}_{\alpha}\left\{u_{1}(x, t)\right\} \\
& -\frac{1}{s^{3 \alpha}}\left\{s^{3 \alpha} u_{1}(s, t)-s^{\alpha} u_{1}^{(\alpha)}(0, t)-\frac{\partial^{3 \alpha} u_{1}(s, t)}{\partial t^{3 \alpha}}\right\} \\
& =\frac{E_{\alpha}\left(t^{\alpha}\right)}{s^{2 \alpha}}+\frac{E_{\alpha}\left(t^{\alpha}\right)}{s^{5 \alpha}}+\frac{E_{\alpha}\left(t^{\alpha}\right)}{s^{8 \alpha}} \\
& =E_{\alpha}\left(t^{\alpha}\right) \sum_{k=0}^{2} \frac{1}{s^{(3 k+2) \alpha}} \\
& \widetilde{L}_{\alpha}\left\{u_{3}(x, t)\right\} \\
& =\widetilde{L}_{\alpha}\left\{u_{2}(x, t)\right\} \\
& -\frac{1}{s^{3 \alpha}}\left\{s^{3 \alpha} u_{2}(s, t)-s^{\alpha} u_{2}^{(\alpha)}(0, t)-\frac{\partial^{3 \alpha} u_{2}(s, t)}{\partial t^{3 \alpha}}\right\} \\
& =\frac{E_{\alpha}\left(t^{\alpha}\right)}{s^{2 \alpha}}+\frac{E_{\alpha}\left(t^{\alpha}\right)}{s^{5 \alpha}}+\frac{E_{\alpha}\left(t^{\alpha}\right)}{s^{8 \alpha}}+\frac{E_{\alpha}\left(t^{\alpha}\right)}{s^{11 \alpha}} \\
& =E_{\alpha}\left(t^{\alpha}\right) \sum_{k=0}^{3} \frac{1}{s^{(3 k+2) \alpha}} \\
& \widetilde{L}_{\alpha}\left\{u_{4}(x, t)\right\} \\
& =\widetilde{L}_{\alpha}\left\{u_{3}(x, t)\right\} \\
& -\frac{1}{s^{3 \alpha}}\left\{s^{3 \alpha} u_{3}(s, t)-s^{\alpha} u_{3}^{(\alpha)}(0, t)-\frac{\partial^{3 \alpha} u_{3}(s, t)}{\partial t^{3 \alpha}}\right\}
\end{aligned}
$$




$$
\begin{aligned}
&= \frac{E_{\alpha}\left(t^{\alpha}\right)}{s^{2 \alpha}}+\frac{E_{\alpha}\left(t^{\alpha}\right)}{s^{5 \alpha}}+\frac{E_{\alpha}\left(t^{\alpha}\right)}{s^{8 \alpha}}+\frac{E_{\alpha}\left(t^{\alpha}\right)}{s^{11 \alpha}}+\frac{E_{\alpha}\left(t^{\alpha}\right)}{s^{14 \alpha}} \\
&= E_{\alpha}\left(t^{\alpha}\right) \sum_{k=0}^{4} \frac{1}{s^{(3 k+2) \alpha}}, \\
& \vdots \\
& \widetilde{L}_{\alpha}\left\{u_{n}(x, t)\right\} \\
&= \widetilde{L}_{\alpha}\left\{u_{n-1}(x, t)\right\} \\
&-\frac{1}{s^{3 \alpha}}\left\{s^{3 \alpha} u_{n-1}(s, t)-\frac{\partial^{3 \alpha} u_{n-1}(s, t)}{\partial t^{3 \alpha}}\right\} \\
&= E_{\alpha}\left(t^{\alpha}\right) \sum_{k=0}^{n} \frac{1}{s^{(2 k+1) \alpha}} .
\end{aligned}
$$

Therefore, the nondifferentiable solution of (29) reads

$$
\begin{aligned}
u(x, t) & =\lim _{n \rightarrow \infty} \widetilde{L}_{\alpha}^{-1}\left\{\widetilde{L}_{\alpha} u_{n}\right\} \\
& =\lim _{n \rightarrow \infty} \widetilde{L}_{\alpha}^{-1}\left\{E_{\alpha}\left(t^{\alpha}\right) \sum_{k=0}^{n} \frac{1}{s^{(3 k+2) \alpha}}\right\} \\
& =E_{\alpha}\left(t^{\alpha}\right) \sum_{k=0}^{n} \frac{x^{(3 k+2) \alpha}}{\Gamma(1+(3 k+2) \alpha)}
\end{aligned}
$$

and its plot is presented in Figure 2.

Example 3. The following local fractional partial differential equation

$$
\frac{\partial^{2 \alpha} u(x, t)}{\partial t^{2 \alpha}}-\frac{\partial^{4 \alpha} u(x, t)}{\partial x^{4 \alpha}}=0
$$

is considered and its initial value is

$$
\begin{array}{rlrl}
\frac{\partial^{3 \alpha} u(0, t)}{\partial x^{3 \alpha}}=E_{\alpha}\left(t^{\alpha}\right), & \frac{\partial^{2 \alpha} u(0, t)}{\partial x^{2 \alpha}}=0, & \frac{\partial^{\alpha} u(0, t)}{\partial x^{\alpha}} & =0, \\
u(0, t) & =0 .
\end{array}
$$

Making use of (19) and (35) the local fractional iteration algorithm reads

$$
\begin{aligned}
& \widetilde{L}_{\alpha}\left\{u_{n+1}(x, t)\right\} \\
&=\widetilde{L}_{\alpha}\left\{u_{n}(x, t)\right\} \\
&-\frac{1}{s^{4 \alpha}} \widetilde{L}_{\alpha}\left\{\frac{\partial^{4 \alpha} u_{n}(x, t)}{\partial x^{4 \alpha}}-\frac{\partial^{2 \alpha} u_{n}(x, t)}{\partial t^{2 \alpha}}\right\} \\
&= \widetilde{L}_{\alpha}\left\{u_{n}(x, t)\right\} \\
&-\frac{1}{s^{4 \alpha}}\left\{s^{4 \alpha} u_{n}(s, t)-s^{3 \alpha} u_{n}(0, t)-s^{2 \alpha} u_{n}^{(\alpha)}(0, t)\right\} \\
&-\frac{1}{s^{4 \alpha}}\left\{-s^{\alpha} u_{n}^{(2 \alpha)}(0, t)-u_{n}^{(3 \alpha)}(0, t)-\frac{\partial^{3 \alpha} u_{n}(s, t)}{\partial t^{3 \alpha}}\right\} \\
&= \widetilde{L}_{\alpha}\left\{u_{n}(x, t)\right\} \\
&-\frac{1}{s^{4 \alpha}}\left\{s^{4 \alpha} u_{n}(s, t)-u_{n}^{(3 \alpha)}(0, t)-\frac{\partial^{3 \alpha} u_{n}(s, t)}{\partial t^{3 \alpha}}\right\},
\end{aligned}
$$

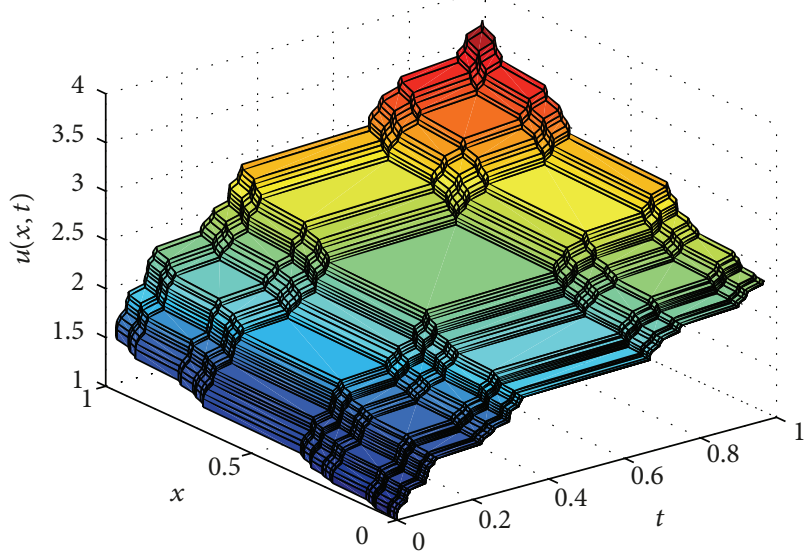

FIGURE 2: Plot of $u(x, t)$ with the parameters $\alpha=\ln 2 / \ln 3$ and $k=$ 50 .

where the initial value is suggested as

$$
\begin{aligned}
\widetilde{L}_{\alpha}\left\{u_{0}(x, t)\right\} & =u_{0}(s, t) \\
& =\widetilde{L}_{\alpha}\left\{\frac{x^{3 \alpha}}{\Gamma(1+3 \alpha)} E_{\alpha}\left(t^{\alpha}\right)\right\}=\frac{E_{\alpha}\left(t^{\alpha}\right)}{s^{4 \alpha}} .
\end{aligned}
$$

From (38) we have the successive approximations as follows:

$$
\begin{aligned}
& \widetilde{L}_{\alpha}\left\{u_{1}(x, t)\right\} \\
&=\widetilde{L}_{\alpha}\left\{u_{0}(x, t)\right\} \\
& \quad-\frac{1}{s^{4 \alpha}}\left\{s^{4 \alpha} u_{0}(s, t)-u_{0}^{(3 \alpha)}(0, t)-\frac{\partial^{3 \alpha} u_{0}(s, t)}{\partial t^{3 \alpha}}\right\} \\
&=\frac{E_{\alpha}\left(t^{\alpha}\right)}{s^{4 \alpha}}+\frac{E_{\alpha}\left(t^{\alpha}\right)}{s^{8 \alpha}} \\
&=E_{\alpha}\left(t^{\alpha}\right) \sum_{k=0}^{1} \frac{1}{s^{4(k+1) \alpha}}, \\
& \widetilde{L}_{\alpha}\left\{u_{2}(x, t)\right\} \\
&=\widetilde{L}_{\alpha}\left\{u_{1}(x, t)\right\} \\
& \quad-\frac{1}{s^{4 \alpha}}\left\{s^{4 \alpha} u_{1}(s, t)-u_{1}^{(3 \alpha)}(0, t)-\frac{\partial^{3 \alpha} u_{1}(s, t)}{\partial t^{3 \alpha}}\right\} \\
&= \frac{E_{\alpha}\left(t^{\alpha}\right)}{s^{4 \alpha}}+\frac{E_{\alpha}\left(t^{\alpha}\right)}{s^{8 \alpha}}+\frac{E_{\alpha}\left(t^{\alpha}\right)}{s^{12 \alpha}} \\
&= E_{\alpha}\left(t^{\alpha}\right) \sum_{k=0}^{2} \frac{1}{s^{4(k+1) \alpha}},
\end{aligned}
$$




$$
\begin{aligned}
& \widetilde{L}_{\alpha}\left\{u_{3}(x, t)\right\} \widetilde{L}_{\alpha}\left\{u_{2}(x, t)\right\} \\
&-\frac{1}{s^{4 \alpha}}\left\{s^{4 \alpha} u_{2}(s, t)-u_{2}^{(3 \alpha)}(0, t)-\frac{\partial^{3 \alpha} u_{2}(s, t)}{\partial t^{3 \alpha}}\right\} \\
&= \frac{E_{\alpha}\left(t^{\alpha}\right)}{s^{4 \alpha}}+\frac{E_{\alpha}\left(t^{\alpha}\right)}{s^{8 \alpha}}+\frac{E_{\alpha}\left(t^{\alpha}\right)}{s^{12 \alpha}}+\frac{E_{\alpha}\left(t^{\alpha}\right)}{s^{16 \alpha}} \\
&= E_{\alpha}\left(t^{\alpha}\right) \sum_{k=0}^{3} \frac{1}{s^{4(k+1) \alpha}}, \\
& \widetilde{L}_{\alpha}\left\{u_{4}(x, t)\right\} \\
&= \widetilde{L}_{\alpha}\left\{u_{3}(x, t)\right\} \\
&-\frac{1}{s^{4 \alpha}}\left\{s^{4 \alpha} u_{3}(s, t)-u_{3}^{(3 \alpha)}(0, t)-\frac{\partial^{3 \alpha} u_{3}(s, t)}{\partial t^{3 \alpha}}\right\} \\
&= \frac{E_{\alpha}\left(t^{\alpha}\right)}{s^{4 \alpha}}+\frac{E_{\alpha}\left(t^{\alpha}\right)}{s^{8 \alpha}}+\frac{E_{\alpha}\left(t^{\alpha}\right)}{s^{12 \alpha}}+\frac{E_{\alpha}\left(t^{\alpha}\right)}{s^{16 \alpha}}+\frac{E_{\alpha}\left(t^{\alpha}\right)}{s^{20 \alpha}} \\
&= E_{\alpha}\left(t^{\alpha}\right) \sum_{k=0}^{4} \frac{1}{s^{4(k+1) \alpha}}, \\
&= \frac{E_{\alpha}\left(t^{\alpha}\right)}{s^{4 \alpha}}+\frac{E_{\alpha}\left(t^{\alpha}\right)}{s^{8 \alpha}}+\frac{E_{\alpha}\left(t^{\alpha}\right)}{s^{12 \alpha}}+\frac{E_{\alpha}\left(t^{\alpha}\right)}{s^{16 \alpha}}+\frac{E_{\alpha}\left(t^{\alpha}\right)}{s^{20 \alpha}} \frac{1}{s^{4(k+1) \alpha}} . \\
& \vdots\left.s^{4 \alpha} u_{n-1}(s, t)-u_{n-1}^{(3 \alpha)}(0, t)-\frac{\partial^{3 \alpha} u_{n-1}(s, t)}{\partial t^{3 \alpha}}\right\} \\
& \widetilde{L}_{\alpha}\left\{u_{n}(x, t)\right\} \\
&=\widetilde{L}_{\alpha}\left\{u_{n-1}(x, t)\right\} \\
&=
\end{aligned}
$$

Hence, the approximate solution of (35) is given by

$$
\begin{aligned}
u(x, t) & =\lim _{n \rightarrow \infty} \widetilde{L}_{\alpha}^{-1}\left\{\widetilde{L}_{\alpha} u_{n}\right\} \\
& =\lim _{n \rightarrow \infty} \widetilde{L}_{\alpha}^{-1}\left\{E_{\alpha}\left(t^{\alpha}\right) \sum_{k=0}^{n} \frac{1}{s^{4(k+1) \alpha}}\right\} \\
& =E_{\alpha}\left(t^{\alpha}\right) \sum_{k=0}^{4} \frac{x^{4(k+1) \alpha}}{\Gamma(1+4(k+1) \alpha)}
\end{aligned}
$$

and its graph is presented in Figure 3.

\section{Conclusions}

Local fractional calculus was successfully applied to deal with the nondifferentiable problems arising in mathematical

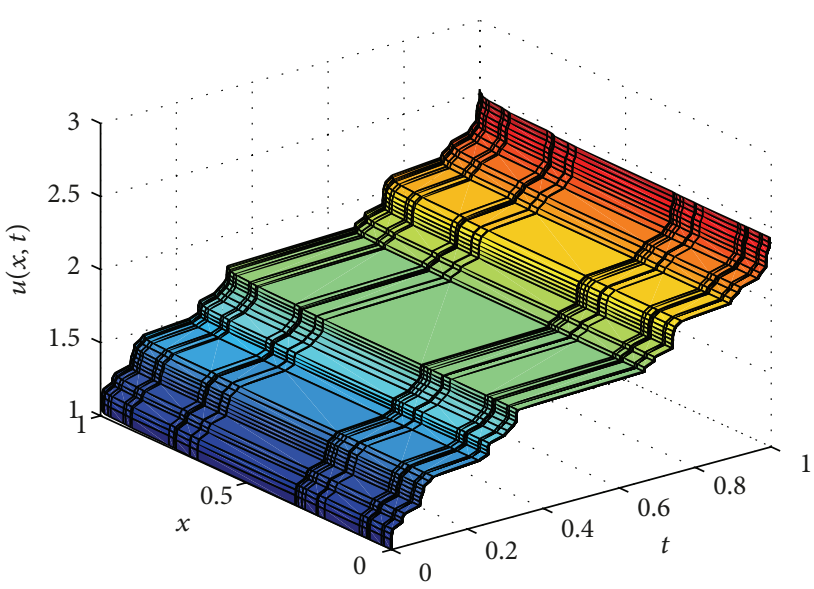

FIGURE 3: Plot of $u(x, t)$ with the parameters $\alpha=\ln 2 / \ln 3$ and $k=$ 50 .

physics. In this work we considered the coupling method of the local fractional variational iteration method and Laplace transform to solve the linear local fractional partial differential equations and their nondifferentiable solutions were obtained. The results are efficient implement of the local fractional Laplace variational iteration method to solve the partial differential equations with local fractional derivative.

\section{Conflict of Interests}

The authors declare that they have no competing interests in this paper.

\section{Acknowledgments}

This work was supported by National Scientific and Technological Support Projects (no. 2012BAE09B00), the National Natural Science Foundation of China (no. 51274270), and the National Natural Science Foundation of Hebei Province (no. E2013209215).

\section{References}

[1] J. Sabatier, O. P. Agrawal, and J. A. T. Machado, Advances in Fractional Calculus: Theoretical Developments and Applications in Physics and Engineering, Springer, Berlin, Germany, 2007.

[2] A. A. Kilbas, H. M. Srivastava, and J. J. Trujillo, Theory and Applications of Fractional Differential Equations, vol. 204 of North-Holland Mathematics Studies, Elsevier Science, Amsterdam, The Netherlands, 2006.

[3] J. Klafter, S. C. Lim, and R. Metzler, Fractional Dynamics: Recent Advances, World Scientific, 2012.

[4] A. H. Bhrawy and D. Baleanu, "A spectral Legendre-GaussLOBatto collocation method for a space-fractional advection diffusion equations with variable coefficients," Reports on Mathematical Physics, vol. 72, no. 2, pp. 219-233, 2013.

[5] A. H. Bhrawy and M. A. Alghamdi, "A shifted Jacobi-GaussLobatto collocation method for solving nonlinear fractional Langevin equation involving two fractional orders in different intervals," Boundary Value Problems, vol. 2012, article 62, 2012. 
[6] J. Hristov, "An exercise with the He's variation iteration method to a fractional Bernoulli equation arising in a transient conduction with a non-linear boundary heat flux," International Review of Chemical Engineering, vol. 4, no. 5, pp. 489-497, 2012.

[7] J. Hristov, "Approximate solutions to fractional subdiffusion equations," European Physical Journal: Special Topics, vol. 193, no. 1, pp. 229-243, 2011.

[8] S. Momani and Z. Odibat, "Analytical solution of a timefractional Navier-Stokes equation by Adomian decomposition method," Applied Mathematics and Computation, vol. 177, no. 2, pp. 488-494, 2006.

[9] Z. Zhao and C. Li, "Fractional difference/finite element approximations for the time-space fractional telegraph equation," Applied Mathematics and Computation, vol. 219, no. 6, pp. 29752988, 2012.

[10] F. Liu, M. M. Meerschaert, R. J. McGough, P. Zhuang, and Q. Liu, "Numerical methods for solving the multi-term timefractional wave-diffusion equation," Fractional Calculus and Applied Analysis, vol. 16, no. 1, pp. 9-25, 2013.

[11] K. M. Kolwankar and A. D. Gangal, "Fractional differentiability of nowhere differentiable functions and dimensions," Chaos, vol. 6, no. 4, pp. 505-513, 1996.

[12] X.-J. Yang, Local Fractional Functional Analysis and Its Applications, Asian Academic Publisher, Hong Kong, 2011.

[13] X.-J. Yang, Advanced Local Fractional Calculus and Its Applications, World Science Publisher, New York, NY, USA, 2012.

[14] G. A. Anastassiou and O. Duman, Advances in Applied Mathematics and Approximation Theory, Springer, New York, NY, USA, 2013.

[15] X.-J. Yang, H. M. Srivastava, J.-H. He, and D. Baleanu, "Cantortype cylindrical-coordinate method for differential equations with local fractional derivatives," Physics Letters A, vol. 377, no. 28-30, pp. 1696-1700, 2013.

[16] X. Yang and D. Baleanu, "Fractal heat conduction problem solved by local fractional variation iteration method," Thermal Science, vol. 17, no. 2, pp. 625-628, 2013.

[17] A. M. Yang, Y. Z. Zhang, and X. L. Zhang, "The nondifferentiable solution for local fractional tricomi equation arising in fractal transonic flow by local fractional variational iteration method," Advances in Mathematical Physics, vol. 2014, Article ID 983254, 6 pages, 2014.

[18] S. P. Yan, H. Jafari, and H. K. Jassim, "Local fractional Adomain decomposition and function decomposition methods for Laplace equation within local fractional operators," Advances in Mathematical Physics, vol. 2014, Article ID 161580, 7 pages, 2014.

[19] A. M. Yang, Z. S. Chen, H. M. Srivastava, and X.-J. Yang, "Application of the local fractional series expansion method and the variational iteration method to the Helmholtz equation involving local fractional derivative operators," Abstract and Applied Analysis, vol. 2013, Article ID 259125, 6 pages, 2013.

[20] Y. Z. Zhang, A. M. Yang, and Y. Long, "Initial boundary value problem for fractal heat equation in the semi-infinite region by Yang-Laplace transform," Thermal Science, vol. 18, no. 2, pp. 677-681, 2014.

[21] C. G. Zhao, A. M. Yang, H. Jafari, and A. Haghbin, “The YangLaplace transform for solving the IVPs with local fractional derivative," Abstract and Applied Analysis, vol. 2014, Article ID 386459, 5 pages, 2014.

[22] S. Wang, Y. J. Yang, and H. K. Jassim, "Local fractional function decomposition method for solving inhomogeneous wave equations with local fractional derivative," Abstract and Applied Analysis, vol. 2014, Article ID 176395, 7 pages, 2014.
[23] C. F. Liu, S. S. Kong, and S. J. Yuan, "Reconstructive schemes for variational iteration method within Yang-Laplace transform with application to fractal heat conduction problem," Thermal Science, vol. 17, no. 3, pp. 715-721, 2013. 


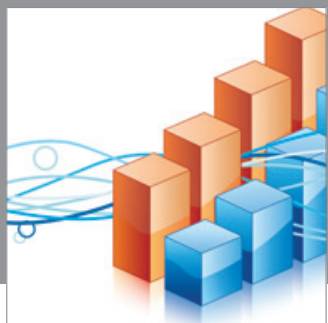

Advances in

Operations Research

mansans

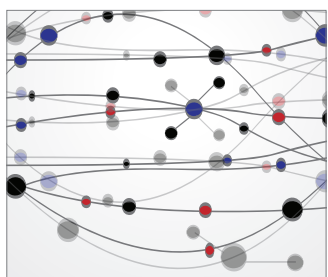

The Scientific World Journal
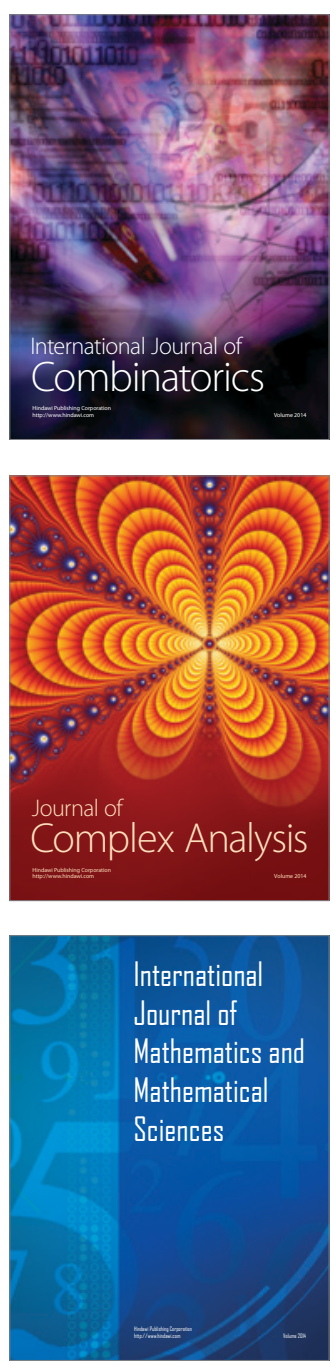
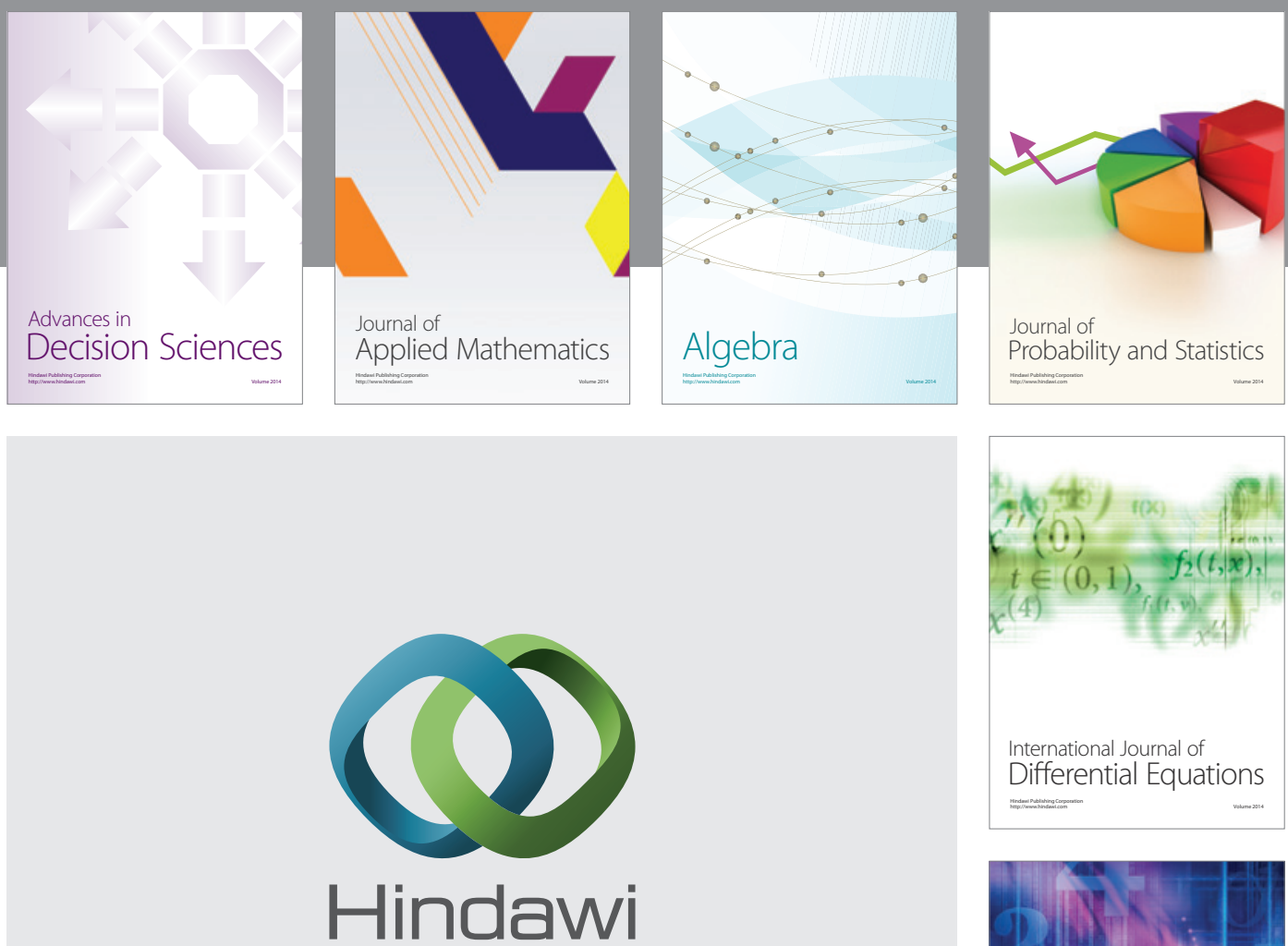

Submit your manuscripts at http://www.hindawi.com
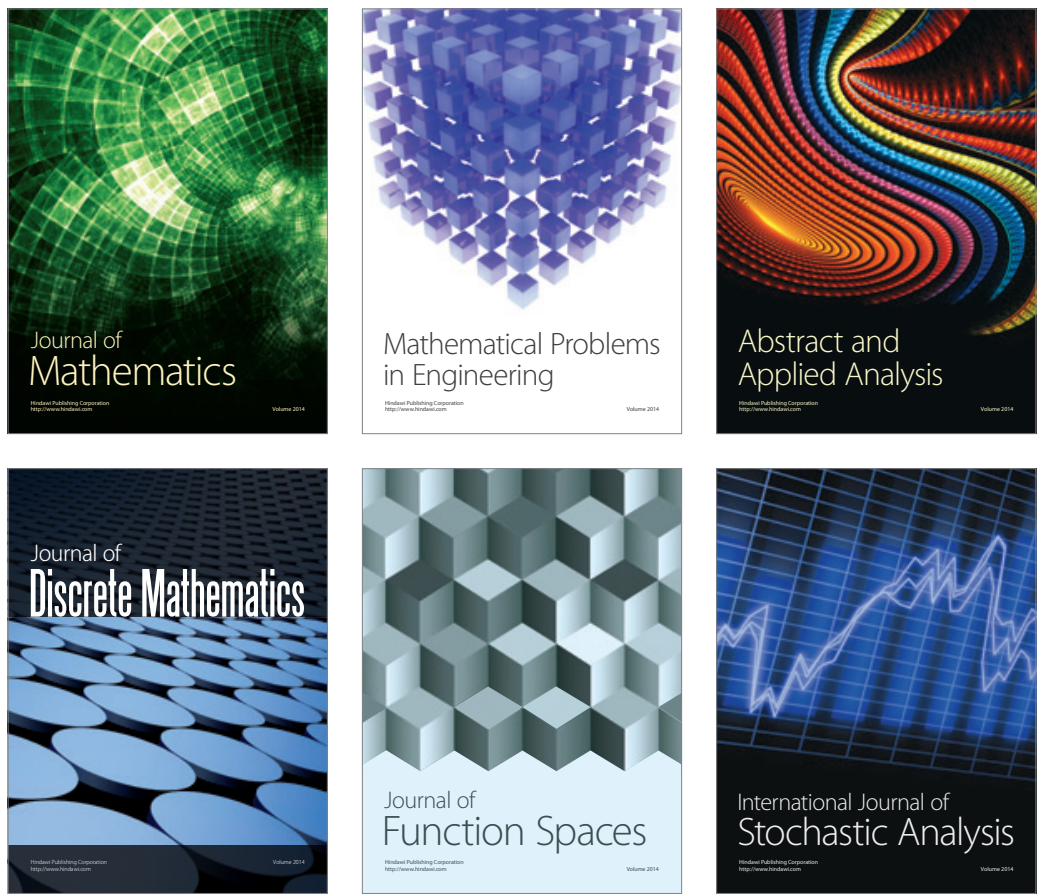

Journal of

Function Spaces

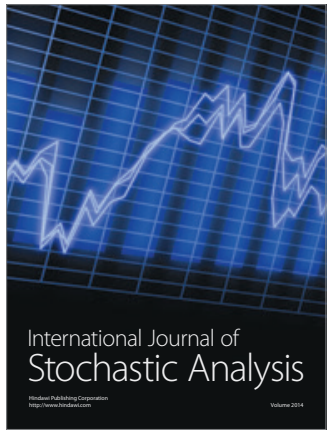

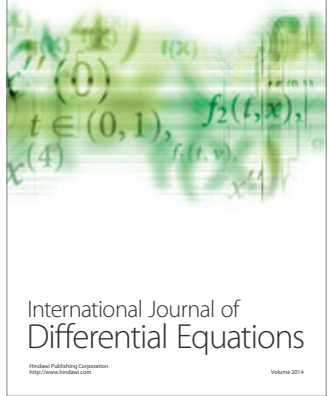
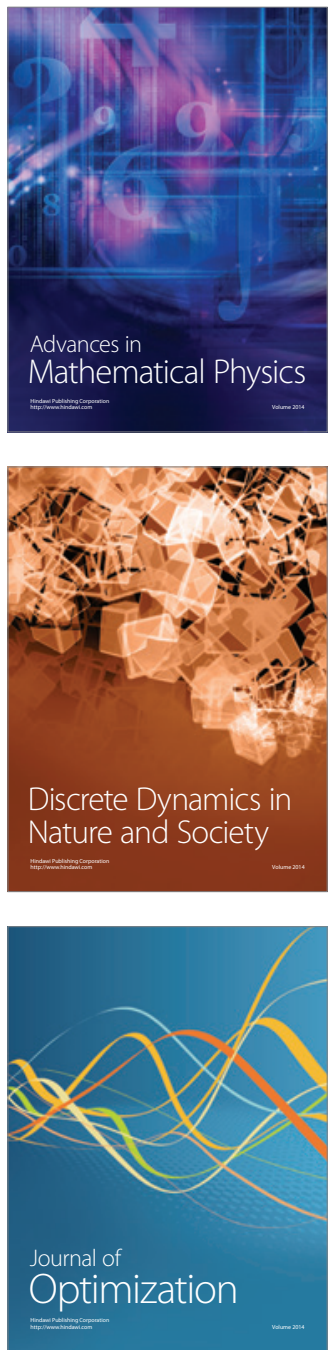